\title{
Assessing the level of confidence for expressing extended uncertainty: a model based on control errors in the measurement of ion activity
}

\author{
Oleksandr Vasilevskyi ${ }^{1}$ \\ ${ }^{1}$ Vinnytsia National Technical University, 95 Khmelnitsky Shose str., 21021, Vinnytsia, Ukraine
}

\section{ABSTRACT}

A method for estimating the level of confidence when determining the coverage factor based on control errors is proposed, using the example of measurements of ion activity. Using information on tolerances and uncertainty, it is possible to establish a reasonable interval around the measurement result, within which most of the values that can be justified are assigned to the measured value.

Section: RESEARCH PAPER

Keywords: measurements; uncertainty; level of confidence; control errors; activity of ions; expanded uncertainty

Citation: Oleksandr Vasilevskyi, Assessing the level of confidence for expressing extended uncertainty through control errors on the example of a model of a means of measuring ion activity, Acta IMEKO, vol. 10, no. 2, article 27, June 2021, identifier: IMEKO-ACTA-10 (2021)-02-27

Section Editor: Maik Rosenberger, Ilmenau University of Technology, Germany

Received March 31, 2020; In final form March 14, 2021; Published June 2021

Copyright: This is an open-access article distributed under the terms of the Creative Commons Attribution 3.0 License, which permits unrestricted use, distribution and reproduction in any medium, provided the original author and source are credited.

Corresponding author: Oleksandr Vasilevskyi, e-mail: o.vasilevskyi@gmail.com

\section{INTRODUCTION}

There is a need to develop methods for estimating the uncertainty of dynamic measurements that meet international requirements for evaluating and expressing the quality of measurements, which is a topical scientific task in the field of metrology. International standards support the use of combined uncertainty $u_{\mathrm{c}}(y)$ as a parameter for quantifying the uncertainty of the measurement result [1]-[4].

Although $u_{\mathrm{c}}(y)$ is widely used to express uncertainty, in some cases, such as in trade, industry, regulatory acts or issues of health and safety, it is advisable to further specify a measure of uncertainty that determines the interval for the measurement result. This additional measure of uncertainty, which corresponds to interval estimation, is called expanded uncertainty.

It is therefore relevant to determine a particular measurement technique to evaluate the trust level for calculating expanded measurement uncertainty.

The purpose of the article is to develop a mathematical apparatus for estimating the confidence level in calculating the expanded uncertainty of measurements of ion activity, taking into account the manufacturer's (developer's) and consumer's control errors, which will make it possible to establish the interval around the measurement result within which the majority of the distribution of values can be attributed to the measured value.

\section{MAIN MATERIALS OF THE RESEARCH}

In the literature [1]-[9], only partial consideration is given to methods of establishing a trust level for calculating the expanded uncertainty of measurement. A mathematical apparatus that would allow a reasonable confidence level in the uncertainty measurement is not described. It is therefore advisable to propose and describe a methodology for estimating trust level based on the control errors of the manufacturer and the consumer, which will make it possible to establish the value of the coefficient of coverage $k$ for the calculation of the expanded uncertainty of measurement. As an example, the developed means was used to measure the activity of ions.

The confidence level for calculating the expanded uncertainty is proposed based on the control errors of the manufacturer and the consumer using the formula

$D=1-P_{n}=1-(\alpha+\beta)$,

Where $\alpha$ represents the control errors of the manufacturer, $\beta$ represents the control errors of the consumer and $P_{n}$ represents the total value of control errors.

The procedure for determining the confidence level is described on the basis of the combined uncertainty of the results 
of reusable measurements of ion activity using a measuring instrument built on the principle of converting voltage to frequency [10], [11], which is described by the following transformation equation:

$N_{\mathrm{U} / \mathrm{F}}=\frac{U_{\text {pow }} f_{0} \tau}{\left(U_{0}-\frac{\alpha_{\mathrm{t}}(273.15+t)}{n_{a}} p X_{i}\right) k_{2}}$

where $p X_{i}$ is the activity of the ions; $\alpha_{\mathrm{t}}$ is the temperature coefficient of steepness, equal to $198.4 \times 10^{-3}{ }^{\circ} \mathrm{C}^{-1}$; $t$ is the temperature of the medium under investigation, $k_{2}$ is the gain scaling factor (DA1 and DA2 in Figure 1), $U_{\text {pow }}$ is the value of the reference voltage of the voltage converter at a frequency of $10 \mathrm{~V} ; \tau=\mathrm{R} C$ is the time constant of the voltage converter at a frequency that is used to set the full scale output frequency range $(\mathrm{R}=1 \mathrm{k} \Omega, C=47 \mu \mathrm{F}), f_{0}$ is the frequency of the quartz resonator microcontroller $(20 \mathrm{MHz}), U_{0}$ is the standard potential of the reference electrode at the initial isopotential point and $n_{a}$ is the charge of the $i^{\text {th }}$ ion [11].

The electrical functional circuit of the ion activity measuring device based on the conversion of voltage to frequency is shown in Figure 1.

The general law of uncertainty when measuring ion activity depends on many factors, such as the activity of interfering ions due to the limited properties of ion-selective electrodes or the presence of measurement error due to temperature, zero drift, instability of the power supply, etc., and it can be difficult to identify which factor is dominant [10]. This allows us to adopt the law of distribution for the centred value of the error when measuring the normal activity of ions, which we describe with the expression

$p(p X)=\frac{1}{u_{c}(p X) \sqrt{2 \pi}} \mathrm{e}^{-\frac{(p X-p \bar{X})^{2}}{2 u_{c}^{2}(p X)}}$

where $p \bar{X}$ is the estimated value of ion activity and $u_{c}(p X)$ is the combined uncertainty of the measurement of ion activity.

The value of the combined uncertainty of ion activity measurement when using a measuring device built on the basis of the conversion of voltage to frequency is calculated by the formula

$u_{c}^{2}(\mathrm{pX})=u_{A}^{2}(p X)+u_{c B}^{2}$

$u_{c B}^{2}=\sum_{i=1}^{N} c_{i}^{2} u_{i}^{2}(p X)+$

$$
+2 \sum_{j=1}^{N-1} \sum_{i=2}^{N} c_{i} c_{j} r(p X, t) u_{i}(p X) u_{j}(t)
$$

where $u_{\mathrm{A}}(p X)$ is the evaluation of type A standard uncertainty, $u_{\mathrm{i}}(p X)$ represents components of type $\mathrm{B}$ uncertainty for measurements of ion activity with the respective sensitivity coefficients $c_{i}, u_{j}(t)$ represents components of type B uncertainty for measurements of temperature with the corresponding sensitivity coefficients $c_{j}$ and $r(p X, t)$ is the correlation coefficient between ion activity $(p X)$ and temperature $(t)$ [10]-[14].

Taking the transformation equation (2) into account, the type $\mathrm{B}$ combined uncertainty is determined by the formula

$u_{c B 1}^{2}=\left(\frac{\partial N_{\mathrm{U} / \mathrm{F}}}{\partial p X_{i}}\right)^{2}\left[u_{B_{E}}^{2}+u_{B_{U / F}}^{2}\right]+$

$$
\begin{aligned}
+ & \left(\frac{\partial N_{\mathrm{U} / \mathrm{F}}}{\partial U_{0}}\right)^{2} u_{B \Theta_{U} 0}^{2}+\left(\frac{\partial N_{\mathrm{U} / \mathrm{F}}}{\partial t}\right)^{2} u_{B_{P}}^{2}+ \\
& +\left(\frac{\partial N_{\mathrm{U} / \mathrm{F}}}{\partial U_{\text {pow }}}\right)^{2} u_{B_{U_{\mathrm{pow}}}}^{2}, \\
u_{c B}^{2}= & u_{c B 1}^{2}+2 \frac{\partial N_{\mathrm{U} / \mathrm{F}}}{\partial p X_{i}} u_{c B 1} \frac{\partial N t_{\mathrm{U} / \mathrm{F}}}{\partial t} u_{c B t} \times \\
& \times \frac{\sum_{l=1}^{n_{i j}}\left(t_{i l}-\overline{t_{i}}\right)\left(p X_{j l}-\overline{p X_{j}}\right)}{\sqrt{\sum_{l=1}^{n_{i j}}\left(t_{i l}-\overline{t_{i}}\right)^{2} \sum_{l=1}^{n_{i j}}\left(p X_{j l}-\overline{p X_{j}}\right)^{2}}}
\end{aligned}
$$

where $N t_{U / F}=\frac{4 U_{\text {pow }} f_{0} \tau}{k_{1} E_{S} \alpha_{t} t}$ is the equation of transformation for measurements of temperature (Figure 1) [10], $E_{\mathrm{s}}=I R_{0}$ is the sensor supply voltage $\left(R_{0}\right.$ is the sensor resistance at a temperature of $0{ }^{\circ} \mathrm{C}$ ) and $k_{1}$ is the gain scaling factor (DA3 in Figure 1),

$$
\frac{\partial N_{\mathrm{U} / \mathrm{F}}}{\partial p X_{i}}=\frac{\alpha_{t}(273.15+t) U_{\mathrm{pow}} f_{0} \tau}{\left[U_{0}-\frac{\alpha_{t}(273.15+t)}{n_{a}} p X_{i}\right]^{2} k_{2} n_{a}}=12.98 p X^{-1}
$$

is the sensitivity factor for ion activity at a temperature of $25^{\circ} \mathrm{C}$,

$$
\frac{\partial N_{\mathrm{U} / \mathrm{F}}}{\partial U_{0}}=-\frac{U_{\mathrm{pow}} f_{0} \tau}{\left[U_{0}-\frac{\alpha_{t}(273.15+t)}{n_{a}} p X_{i}\right]^{2} k_{2}}=-0.22 \mathrm{~V}^{-1}
$$

is the coefficient of the voltage sensitivity of the standard potential of the reference electrode,

$$
\frac{\partial N_{\mathrm{U} / \mathrm{F}}}{\partial t}=\frac{\alpha_{t} p X_{i} U_{\mathrm{pow}} f_{0} \tau}{\left[U_{0}-\frac{\alpha_{t}(273.15+t)}{n_{a}} p X_{i}\right]^{2} k_{2} n_{a}}=0.3^{\circ} \mathrm{C}^{-1}
$$

is the coefficient of sensitivity at an additional measured temperature,

$$
\frac{\partial N_{\mathrm{U} / \mathrm{F}}}{\partial U_{\mathrm{pow}}}=\frac{f_{0} \tau}{\left(U_{0}-\frac{\alpha_{t}(273.15+t)}{n_{a}} p X_{i}\right) k_{2}}=-9.08 \mathrm{~V}^{-1}
$$

is the coefficient of the voltage sensitivity of the stable power supply source of the voltage-to-frequency converter (VFC), $n_{i j}$ is the number of pairwise measurements of temperature and ion activity, $u_{B_{E}}=\gamma \frac{p X_{\max }}{100 \% \sqrt{3}^{-3}} p X$ is the type $\mathrm{B}$ uncertainty caused by the presence of a primary electrode with a low accuracy class $(\gamma=0.7 \%), u_{B \Theta_{U 0}}=\frac{\Theta_{U 0}}{\sqrt{3}}=0.95 \mathrm{mV}$ is the type $\mathrm{B}$ uncertainty caused by the instability $\left(\Theta_{U 0}=1.65 \mathrm{mV}\right)$ of the standard potential of the reference electrode, $u_{B p}=\frac{\Delta \text { Ubias }_{\max }}{U_{\text {Pout }} \sqrt{3}} \approx 2.89$. $10^{-3}{ }^{\circ} \mathrm{C}$ is the type $\mathrm{B}$ uncertainty caused by the existence of a bias voltage $\left(\right.$ Ubias $_{\text {max }} / U_{\text {Pout }}=0.2 \cdot 10^{-3}$ ) of the operational amplifier when the temperature deviates $\left(\Delta t=5{ }^{\circ} \mathrm{C}\right)$ from the nominal value, $u_{B_{U_{\mathrm{POW}}}}=\frac{\Theta_{U_{P}}}{\sqrt{3}} \approx 1.16 \mathrm{mV}$ is the type $\mathrm{B}$ uncertainty caused by instability in the reference voltage power supply source $\left(\Theta_{U_{P}}=2 \mathrm{mV}\right)$ and $u_{B_{\mathrm{VFC}}}=\frac{\delta_{\text {nonl }} p X_{\max }}{100 \% \sqrt{3}^{-6}} p X$ is the type B uncertainty caused by the nonlinearity $\left(\delta_{\text {nonl }}\right)$ of the voltage converter (AD650) [10]-[14].

The combined uncertainty values are calculated in [10] and [14], and the maximum uncertainty value for measuring the 
activity of phosphate ions is $17.21 \times 10^{-3} p X$ in the range of measurements from 6 to $0.3 p X$.

A compatible two-dimensional confidence level density when measuring the activity of ions, taking into account the allowable deviation of the measurement error $\varepsilon$, which is established by the consumer, is described by the expression

$p(p X, \varepsilon)=\frac{1}{2 \pi \cdot u_{c}(p X) \cdot u_{\varepsilon}} \mathrm{e}^{-\frac{(p X-p \bar{X})^{2}}{2 u_{c}^{2}(p X)}-\frac{(\Delta \varepsilon)^{2}}{2 u_{\varepsilon}^{2}}}$.

In most practical cases, as noted in [9] and [15], the admissible deviation $\varepsilon$ of the parameter controlled by the user, depending on the combined uncertainty of the measurement results $u_{c}(y)$, is determined by the formula

$\varepsilon[\%]=\frac{6 u_{c}(y)}{d} 100=\frac{6 u_{c}(y)}{X_{\max }-X_{\min }} 100$,

where $d$ is the width of the tolerance field determined by the values of the upper $X_{\max }$ and lower $X_{\min }$ range of measurement for the physical quantity.

By substituting the values of the maximum combined uncertainty when measuring ion activity $u_{\mathrm{c}}(p X)=17.21 \cdot 10^{-3} p X$ and the values of the upper $\left(X_{\max }=6 p X\right)$ and the lower $\left(X_{\min }=\right.$ $0.3 p X)$ ranges of the measurement limit in equation (9), we obtain the value of the permissible error set by the consumer, which is $\varepsilon=1.81 \%$.

The uncertainty $u_{\varepsilon}$ of the tolerance field of the controlled parameter at which the result can be considered reliable in practice is recommended to be equal to $6 u_{\mathrm{c}}(y)$ [9], [13], [15]-[18]. Thus, $u_{\varepsilon}=6 \times u_{\mathrm{c}}(y)=6 \times 17.21 \cdot 10^{-3}=103.26 \cdot 10^{-3} p X$.

Taking into account expression (8), the manufacturer's control errors $\alpha$ are estimated by the formula

$$
\begin{array}{r}
\alpha=\int_{X_{\min }}^{X_{\max }}\left[\int_{-\infty}^{X_{\min }} \frac{\mathrm{e}^{-\frac{(\Delta p X)^{2}}{2 u_{c}^{2}(p X)}-\frac{(\Delta \varepsilon)^{2}}{2 u_{\varepsilon}^{2}}}}{2 \pi \cdot u_{c}(p X) \cdot u_{\varepsilon}}+\right. \\
+\int_{X_{\max }}^{\infty} \frac{\mathrm{e}^{-\frac{(\Delta p X)^{2}}{2 u_{c}^{2}(p X)}-\frac{(\Delta \varepsilon)^{2}}{2 u_{\varepsilon}^{2}}}}{2 \pi \cdot u_{c}(p X) \cdot u_{\varepsilon}} d \Delta \varepsilon
\end{array}
$$

and the consumer's control errors $\beta$ are estimated by the formula

$$
\begin{aligned}
\beta= & \int_{-\infty}^{X_{\min }} \int_{X_{\min }+\Delta p X}^{X_{\max }-\Delta p X} \frac{\mathrm{e}^{-\frac{(\Delta p X)^{2}}{2 u_{c}^{2}(p X)}-\frac{(\Delta \varepsilon)^{2}}{2 u_{\varepsilon}^{2}}}}{2 \pi \cdot u_{c}(p X) \cdot u_{\varepsilon}} d \Delta p X d \Delta \varepsilon+ \\
+ & \int_{X_{\max }}^{\infty} \int_{X_{\min }+\Delta p X}^{X_{\max }-\Delta p X} \frac{\mathrm{e}^{-\frac{(\Delta p X)^{2}}{2 u_{c}^{2}(p X)}-\frac{(\Delta \varepsilon)^{2}}{2 u_{\varepsilon}^{2}}}}{2 \pi \cdot u_{c}(p X) \cdot u_{\varepsilon}} d \Delta p X d \Delta \varepsilon .
\end{aligned}
$$

The admission field is the value of the measured value $\Delta p X$. In this case, this is the activity of the $p X$ ions, which is defined by the formula

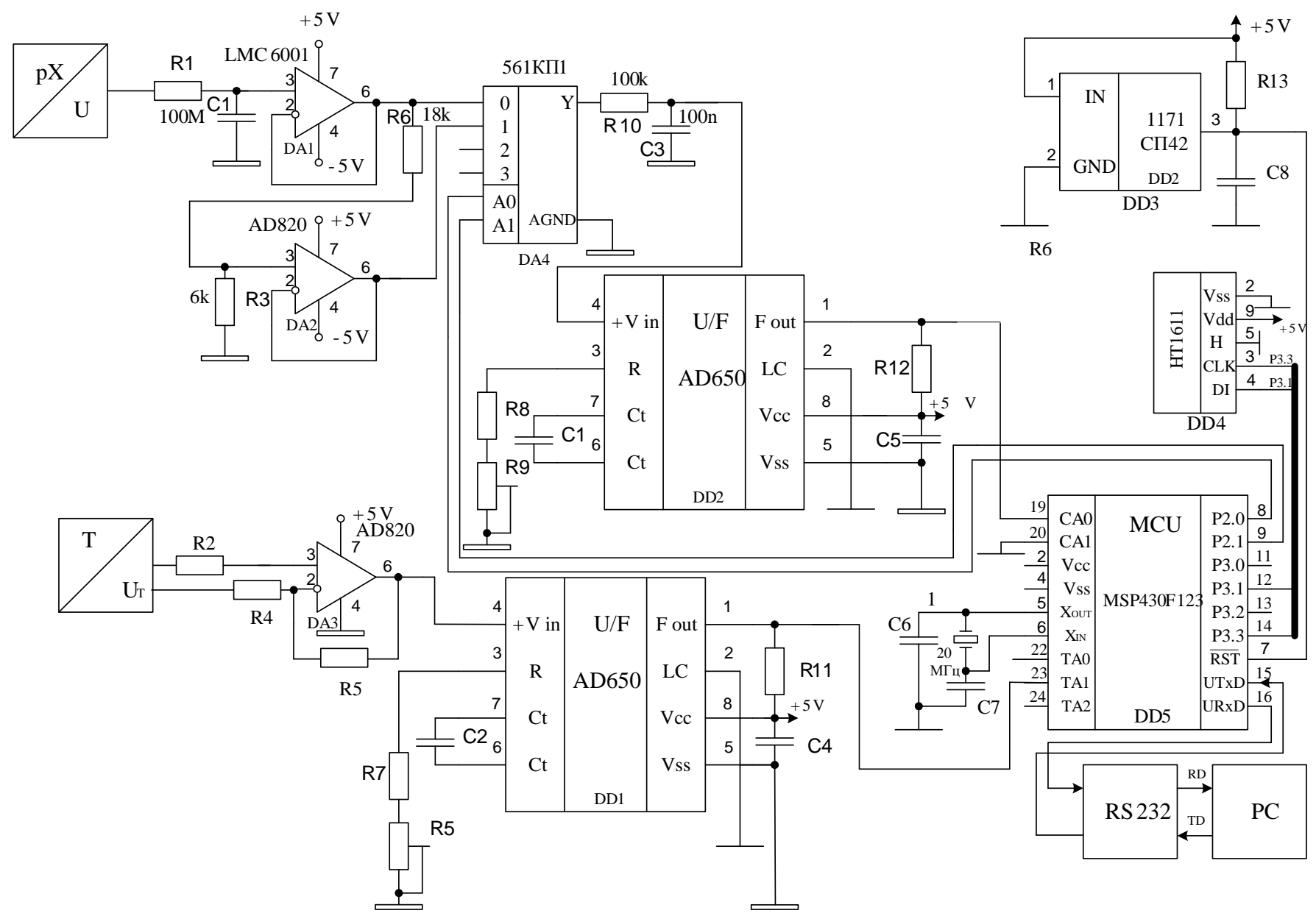

Figure 1. The functional electrical circuit of the device for measuring ion activity. 
$\Delta p X=\frac{p \bar{X}}{100} \delta_{\max }$,

where $p \bar{X}$ is the estimation of the ion activity in the upper measurement range, $\delta_{\max }$ is the maximum relative measurement error (for measurements of ion activity, this value is $0.7 \%$ in the range of measurements from 6 to $0.3 p X$ ).

Consequently, when calculating the control errors of the manufacturer and the consumer, the field of admission $\Delta p X$ can be calculated by (12), which, with the corresponding numerical values, is equal to $2.1 \cdot 10^{-3} p X$.

By substituting calculated tolerances in the formula for estimating the control errors of the manufacturer (10) and the consumer (11) and solving them using the Maple 12 package, we obtain the following numerical values: $\alpha=0.24 \cdot 10^{-67}(\alpha \approx 0)$, and $\beta=0.00196$. The total value of the control errors is $P_{n}=$ 0.00196 , and the confidence level for calculating the expanded uncertainty of the measurement, according to formula (1) will be equal to $D=1-P_{n}=1-0.00196=0.998$.

Figure 2 illustrates the change in the manufacturer's and consumer's control errors depending on the parameter $\mu$, which is equal to $u_{\varepsilon} / u_{\mathrm{c}}(p X)$. This parameter establishes the relationship between the permissible uncertainty of $u_{\varepsilon}$, which is specified by the consumer (via normative documents), and the combined uncertainty of $u_{\mathrm{c}}(p X)$, which is set by the manufacturer. The figure shows $u_{\varepsilon}<u_{\mathrm{c}}(p X), u_{\varepsilon} \approx u_{\mathrm{c}}(p X)$ and $u_{\varepsilon}>u_{\mathrm{c}}(p X)$.

Thus, based on the constructed characteristic of the change in reliability (Figure 2), which is obtained by calculating the consumer and manufacturer's control errors, a range can be calculated within which the majority of the distribution of values that can be attributed to the measured value is likely to be located, depending on the value of the accepted tolerance for the monitored parameter.

As can be seen from Figure 2, with a tolerance of six combined uncertainties, the confidence level is $99.8 \%$.
Given the level of confidence based on control errors, the expanded uncertainty can be calculated from the formula

$U=k_{p} \cdot u_{c}(y)=k_{99.8} \cdot 17.21 \cdot 10^{-3}$,

where $k_{p}$ is the coverage ratio for the established confidence level $(p=D)$, which is taken from the Student's table.

Thus, with 30 degrees of freedom and a confidence probability of $99.8 \%$, the coverage factor $k_{p}$ is 3.385 , and with 10 degrees of freedom and a probability of $99.8 \%$, it is 4.14 . Accordingly, if these coverage factor values are entered into (13), the expanded uncertainty will be $U_{99.8}=71.25 \cdot 10^{-3} p X$ for 10 degrees of freedom and $U_{99.8}=58.26 \cdot 10^{-3} p X$ for 30 degrees of freedom.

\section{CONCLUSIONS}

The work describes the characteristics of the change in the manufacturer's and consumer's control errors, on the basis of which a general characteristic of the change in the control errors that occurs during the measurement of ion activity is obtained. Using this characterisation, it is possible to determine graphically the confidence level within which most of the distribution of values obtained by measuring the activity of ions is probably located.

The obtained nomograph makes it possible to determine with a high probability the confidence level at a given tolerance for the controlled parameter to determine the coverage factor in calculating the extended uncertainty of ion activity measurement.

The described approach for determining confidence probability on the basis of control errors can be applied to any type of measurements, provided a separate calculation is performed to characterise the control errors of the manufacturer of the measuring instrument and the consumer (i.e. errors of the first and second kind).

D

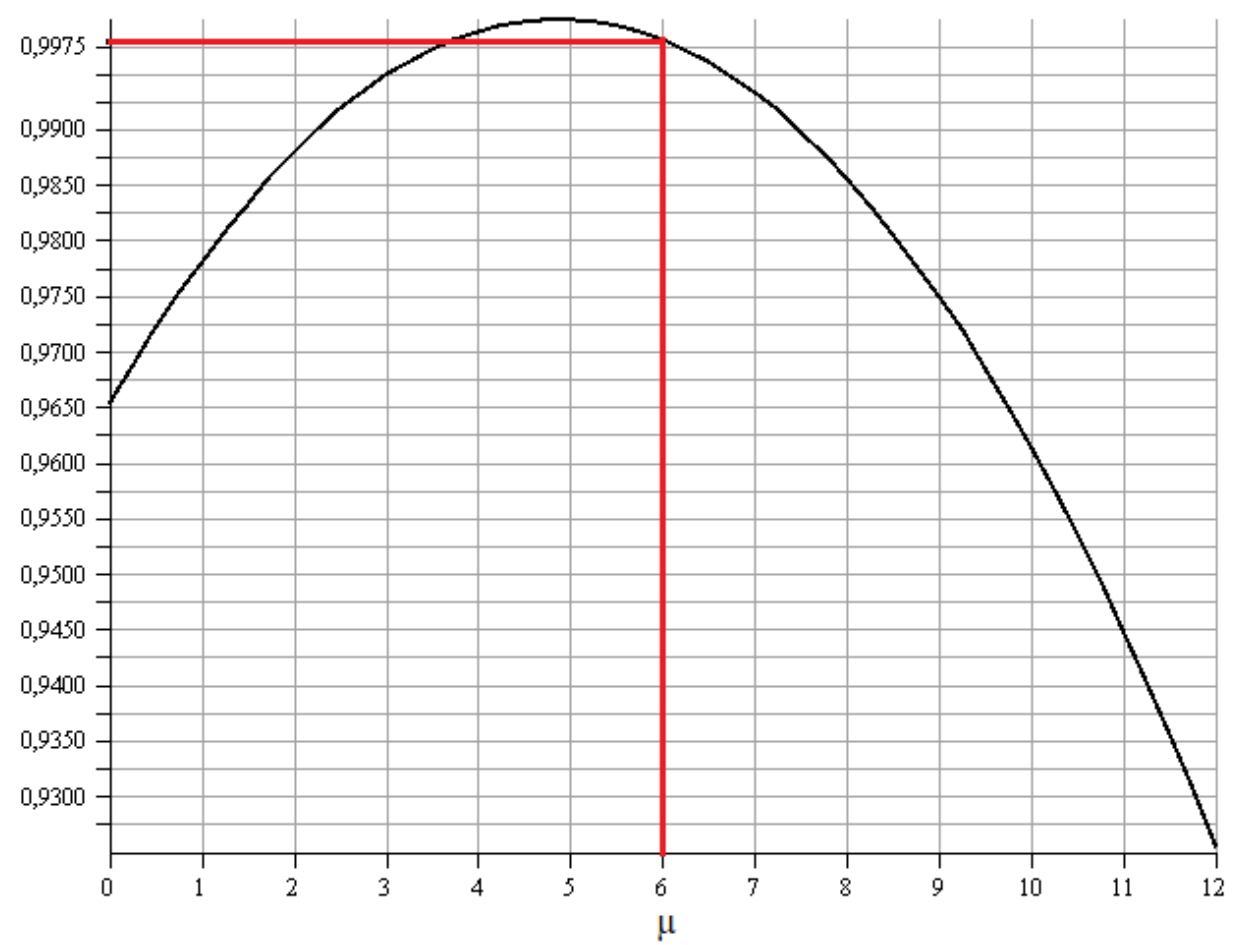

Figure 2. Characteristics of the change of control errors depending on the parameter $\mu$. 


\section{REFERENCES}

[1] BIPM, IEC, IFCC, ISO, IUPAC, IUPAP, OIML, Evaluation of measurement data - guide to the expression of uncertainty in measurement, Joint Committee for Guides in Metrology, Bureau International des Poids et Mesures JCGM 100:2008 (2008).

[2] BIPM, IEC, IFCC, ISO, IUPAC, IUPAP, OIML, Evaluation of measurement data - supplement 2 to the 'Guide to the expression of uncertainty in measurement' - extension to any number of output quantities, Joint Committee for Guides in Metrology, Bureau International des Poids et Mesures JCGM 102:2011 (2011).

[3] BIPM, IEC, IFCC, ISO, IUPAC, IUPAP, OIML, Evaluation of measurement data - an introduction to the 'Guide to the expression of uncertainty in measurement' and related documents, Joint Committee for Guides in Metrology, Bureau International des Poids et Mesures JCGM 104:2009 (2009).

[4] ISO/IEC, Uncertainty of measurement - part 1: introduction to the expression of uncertainty in measurement, ISO/IEC Guide 98-1:2009 (2009).

[5] O. M. Vasilevskyi, Calibration method to assess the accuracy of measurement devices using the theory of uncertainty, Int. J. Metrol. Qual. Eng. 5(4) (2014) 403, 9 pp.

DOI: $10.1051 /$ ijmqe/2014017

[6] IEC, Application of uncertainty of measurement to conformity assessment activities in the electrotechnical sector, IEC GUIDE 115-2007 (2007).

[7] ISO/IEC, General requirements for the competence of testing and calibration laboratories, ISO/IEC 17025:2005 (2005).

[8] O. M. Vasilevskyi, Methods of determining the recalibration interval measurement tools based on the concept of uncertainty, Technical Electrodynamics 6 (2014) pp. 81-88.

[9] S. F. Beckert, W. S. Paim, Critical analysis of the acceptance criteria used in measurement systems evaluation, Int. J. Metrol. Qual. Eng. 8 (2017) art. 23. DOI: $10.1051 /$ ijmqe/2017016

[10] O. M. Vasilevskyi, V. M. Didych, Elements of the Theory of Constructing Potentiometric Means of Measuring Control of Ion Activity with an Increased Probability [Monograph], Vinnytsia: VNTU, 2013. Online [Accessed 2 June 2021] http://vasilevskiy.vk.vntu.edu.ua/ file/d23a8f23c4fdc081c30ebb0 9bc7182e6.pdf

[11] V. M. Didych, O. M. Vasilevskyi, V. O. Podzharenko, Potentiometer facilities of ions activity measurement of humus elements in soil, Visnyk of Vinnytsia Politechnical Institute 5 (2008) pp. 5-10. Online [Accessed 2 June 2021] https://visnyk.vntu.edu.ua/index.php/visnyk/article/view/639/ $\underline{638}$
[12] O. M. Vasilevskyi, Metrological characteristics of the torque measurement of electric motors, Int. J. Metrol. Qual. Eng. 8 (2017) 7, 9 pp. DOI: $10.1051 /$ ijmqe $/ 2017005$

[13] O. M. Vasilevskyi, V. M. Didych, A. Kravchenko, M. Yakovlev, I. Andrikevych, D. Kompanets, Y. Danylyuk, W. Wójcik, A. Nurmakhambetov, Method of evaluating the level of confidence based on metrological risks for determining the coverage factor in the concept of uncertainty, Proceedings Volume 10808, Photonics Applications in Astronomy, Communications, Industry, and High-Energy Physics Experiments, WUT Wilga Resort, Poland, 2 - 10 June 2018.

DOI: $10.1117 / 12.2501576$

[14] O. M. Vasilevskyi, V. Y. Kucheruk, V. V. Bogachuk, K. Gromaszek, W. Wójcik, S. Smailova, N. Askarova, The method of translation additive and multiplicative error in the instrumental component of the measurement uncertainty, Proc. SPIE 10031, Photonics Applications in Astronomy, Communications, Industry, and High-Energy Physics Experiments, WUT Wilga Resort, Poland, 29 May - 6 June 2016. DOI: $10.1117 / 12.2249195$

[15] D. J. Wheeler, An honest gauge R\&R study, 2006 ASQ/ASA Fall Technical Conference, Columbus, US, 12 - 13 October 2006. Online [Accessed 2 June 2021] http://www.spcpress.com/pdf/DJW189.pdf

[16] O. M. Vasilevskyi, P. I. Kulakov, I. A. Dudatiev, V. M. Didych, A. Kotyra, B. Suleimenov, A. Assembay, A. Kozbekova,

Vibration diagnostic system for evaluation of state interconnected electrical motors mechanical parameters, Proc. SPIE 10445, Photonics Applications in Astronomy, Communications, Industry, and High Energy Physics Experiments, WUT Wilga Resort, Poland, 29 May - 6 June 2016. DOI: $10.1117 / 12.2280993$

[17] O. M. Vasilevskyi, V. Y. Kucheruk, V. V. Bogachuk, K. Gromaszek, W. Wójcik, S. Smailova, N. Askarova, The method of translation additive and multiplicative error in the instrumental component of the measurement uncertainty, Proc. SPIE 10031, Photonics Applications in Astronomy, Communications, Industry, and High-Energy Physics Experiments, WUT Wilga Resort, Poland, 29 May - 6 June 2016. DOI: $10.1117 / 12.2249195$

[18] V. O. Podzharenko, O. M. Vasilevskyi, Diagnostics of technical condition of electromechanical systems for the logarithmic decrement, Proceedings of Donetsk National Technical University (2005). 